\title{
How weather affects energy demand variability in the transition towards sustainable heating
}

Sven Eggimann ${ }^{* 1,2}$, Will Usher ${ }^{1,3}$, Nick Eyre ${ }^{l}$, and Jim W. Hall ${ }^{1}$

1Environmental Change Institute, University of Oxford, South Parks Road, Oxford OXI 3QY, UK. 2Urban Energy Systems Laboratory, Swiss Federal Laboratories for Materials Science and Technology, Empa, Dübendorf, Switzerland. 3Division of Energy System Analysis, KTH Royal Institute of Technology, Stockholm, Sweden

*Corresponding author: sven.eggimann@empa.ch

\begin{abstract}
Electrification of heat will impact demands on power systems, potentially increasing sensitivity to weather variability. We have developed a spatio-temporal methodology for assessing electricity demand in the context of weather variability, with a particular focus upon electrification of heat. We apply our model to analyse varying levels of electrification of heat in the United Kingdom and simulate local weather impacts with an ensemble of 100 weather realisations. Across the scenarios, the maximum simulated national electricity peak demand doubles compared to today. Assuming current weather pattern, the weather-induced variability in electricity peak is projected to range from $6.1-7.8 \mathrm{GW}(10.2-15.2 \%$ of mean peak demand) in 2020 to $6.2-14.6 \mathrm{GW}$ (9.8-22.2\% of mean peak demand) in 2050. We find that future weather may exacerbate the impact of electrification of heat on peak demand. However, socio-economic uncertainty predominates weather-induced variability. Electrification of heat without reducing heating demands will result in dramatic increases in peak electricity demand as well as increased exposure to weather effects. Regions experiencing a combined increase in peak demand and weather variability will likely prove to be particularly challenging for balancing demand and supply. Decarbonizing heat by switching to alternatives fuels such as hydrogen or measures to lower heating demand reduces the need for additional peak electricity capacity as well as mitigating impacts of extreme weather events.
\end{abstract}

\section{Keywords}

decarbonisation; energy transition; scenario analysis; geospatial modelling; hydrogen; electrification 


\section{Introduction}

In many countries, the existing carbon-intensive heating infrastructure needs to undergo a dramatic transformation to transition to a low-carbon energy system and to achieve emission targets (Unruh, 2000; Foxon, 2013; Markard, 2018). Previous studies have identified how low carbon heating may be achieved by decarbonisation of the electricity supply sector combined with electrification of heating(Barton et al., 2013; Eyre and Baruah, 2015). Escaping the lockin of carbon-based fuels is particularly difficult for countries such as the United Kingdom (UK) for which heating is a large of primary energy consumption and depend predominantly on carbon-based fuels such as natural gas and oil to deliver this. Whereas the decarbonisation literature demonstrates simultaneous decarbonisation by electrification of heat and transport (inter alia Jalil-Vega and Hawkes, 2018a; Baruah et al., 2014; Qadrdan et al., 2015), existing energy demand projections are only starting to include the changing system exposures to other factors such as weather when considering long-term uncertainties in drivers of energy demands (inter alia Zeyringer et al. 2018, Clegg and Mancarella 2019, and Watson et al. 2019). This is particularly true for simulation models projecting energy demands at a high temporal and spatial scale. Electrification of heat is however expected to place additional operational requirements due to future changes in peak electricity demands as well as changes to exposure to weather variability, on a regional as well as on a national scale.

Exactly how future national and regional energy demands will develop is however uncertain. The evolution of future energy demands depends on a range of technical, socio-economic, environmental and behavioural factors. High levels of electrification and substantial decarbonisation will imply behavioural, policy and economic challenges (Eyre et al., 2018). For the United Kingdom, in order to achieve emission targets, gas will need to be substituted by alternative fuel types (Chaudry et al., 2015) and this necessitates the deployment of technologies such as heat pumps, although the deployment of these is lagging behind other countries (Hannon, 2015).

So far, future uncertainties in UK energy demands have been explored in various studies, predominantly projecting energy demands on a national and annual scale (Eyre and Baruah, 2015; Skea et al., 2011; National Grid, 2017). Only recently have energy demand simulations included combined high spatial and temporal resolutions (Schneider et al., 2017; Clegg and Mancarella, 2019; Jalil-Vega and Hawkes, 2018a; Jalil-Vega and Hawkes, 2018b). Resolving spatial and temporal characteristics of energy demand is however important as energy transitions are spatial (Truffer, 2012; Morton et al., 2018). The spatial nature of changes of drivers of energy demand such as changing population dynamics or changes in economic activity will lead to new energy demand geographies (Bridge et al., 2013). Simulating at a high spatial and temporal resolution also enables the investigation of key environmental drivers of energy demand, such as climate and weather, which typically are considered only by building energy models and gas supply models, but not in a whole-energy system setting. However, high-resolution modelling raises its own set of challenges, such as limited data availability and high necessary computational power (Pfenninger et al., 2014). In particular, incorporating local 
weather information is essential for high-resolution modelling of heating demand. With a few exceptions (inter alia Staffell and Pfenninger, 2018), weather related impacts are generally neglected in high spatio-temporal energy demand projections. Information on weather-induced variability can inform policy making in various ways. For example, spatial knowledge about future changes in peak capacity due to weather effects can influence the adoption of effective demand side management policies and the planning of energy infrastructures such as network reinforcement. Identifying regions with a combined increase in variability and demand will need special consideration and would benefit most from policies to reduce weather related effects. Even though electricity and gas demands may be supplied from anywhere when connected across a transmission network, within operating constraints, high-resolution knowledge about regional energy demand allows operators to plan for network reinforcements, in case substations or lower voltage lines will not be able to handle additional future power flows (Burillo et al., 2019). In the case of more decentralised energy systems placing higher demands on local generation, such as the adoption of mini-grids in many developing countries, matching energy demand and supply at local-scales will become increasingly important. Local communities will rely more and more on balancing local energy demand and supply and high-resolution information is necessary for incorporating renewable generation.

In this paper, we develop a highly resolve spatio-temporal model of energy demand and use it to explore long-term energy demand variability in the United Kingdom with respect to different socio-economic scenarios and degrees of electrification. To study the effect of weatherinduced variability, regionally explicit temperatures are included: regional electricity peak demand is simulated for an ensemble of 100 different temperature profiles drawn from the United Kingdom specific weather microsimulation of the RCP8.5 climate scenario. This is the highest RCP scenario, allowing the investigation of a situation where global mitigation efforts fail and notable change to weather. As a result, we identify regions with anticipated high changes in demand which coincide with high exposures to weather related variability. In Section 2.1, the applied energy demand simulation framework is explained. Section 2.2 outlines how weather variability is modelled. Section 2.3 introduces the energy demand scenarios, Section 3 presents national as well as regional high-resolution modelling results and discusses potential implications. Key conclusions with respect to potential policy implications are presented in Section 4.

\section{Method}

\subsection{High-resolution energy demand modelling}

A combined spatial and temporal high-resolution energy demand simulation model is used to project end-use specific energy demands for the United Kingdom. The used energy demand model has been developed within the context of the MISTRAL program (Hall et al. 2016, https://www.itrc.org.uk/), which has the goal of simulating interdependent infrastructure sectors, including energy, transport, digital communications and water. The energy demand model allows the simulation of long-term changes in residential, service and industrial energy demands on an hourly scale and for all local authority districts across the United Kingdom. 
The model is applied for the simulation of innovative technologies and policies, and their diffusion across space and time. It allows the consideration of socio-technical trends and simulates energy demand for different fuel types such as gas, electricity or hydrogen for alternative futures. The model is based on a decomposition approach, where national energy demand statistics developed by BEIS (2018) are temporally and spatially disaggregated for the simulation base year 2015 into 28 end-uses, 34 sectors and 7 fuel types. The main methodological steps can be summarized as follows: Firstly, national energy demands are spatially disaggregated to local authority district level (NUTS 3) using population as a proxy. Secondly, regional annual end-use specific energy demands are temporally disaggregated to hourly demands, where either technology or demand specific load profiles are used from different measurement trial data sets. Specifically, for simulating heating demands, daily heating demands are simulated by calculating daily Heating Degree Days (HDD) (see Section 2.2). The disaggregated base year service and industry energy demands are calibrated with the help of non-residential gas and electricity demands taken from BEIS (2018). Thirdly, energy demands are projected with scenario data for each simulation year for each end-use following a backcasting approach. The fully open-source model is described in full detail in Eggimann et al. (2019).

\subsection{Modelling energy demand for heating}

The end uses of heating and cooling are of primary interest to study the effect of weather variability and a changing climate on energy demands as they are a function of temperature and other weather variables. In the United Kingdom, the majority of energy used for thermal comfort is concentrated in the winter months, when outside temperatures dip below comfortable levels for extended periods. Consequently, variation in temperature will drive variation in demand for heating (Thornton et al., 2016). In colder climates, the impact of weather on heating buildings is generally greater (Hong et al., 2013), even though heating is not only a function of weather but also of other factors such as building insulation levels. For the United Kingdom, the demand for space and water heating is substantial ( $\sim 34 \%$ of overall final energy demand and $\sim 58 \%$ excluding transportation for the year 2017).

For modelling heating demands, the Heating Degree Day (HDD) is a commonly used measure, due to its good correlation with actual energy consumption for heating (Cox et al., 2015; Fikru and Gautier, 2015; Spoladore et al., 2016). HDD are particularly suited for modelling at a high temporal and spatial resolution as they depend on hourly temperatures and thus capture the variability in spatially explicit weather effects by incorporating spatial variation in temperature given that a high enough resolution of weather data is used (Department of the Environment, 1993). Using only a small number of representative weather stations may lead to biases in modelled peak demand as shown by Burleyson et al. (2017). For our study, we use over 200 weather stations distributed across the United Kingdom. For each of the 391 modelled regions (local authority districts), we use the geographically closest weather station for calculating regional HDD. Potential temperature variation within a region is ignored. Recognising that the relationship between temperature and energy demands is country specific (Gallo Cassarino et al., 2018), for the United Kingdom, we simulate the change in heating demand based on an assumed linear relationship between the number of HDD and heating demand. As shown for example by Sansom (2014), assuming a linear relationship between HDD and 
change in heating demand is a good approximation. HDD are calculated from of a base temperature assumption of $15.5 \mathrm{C}$, which is commonly assumed for the United Kingdom (Carbon Trust, 2012). Because the calculation of HDD is directly linked to temperatures, HDD are also directly related to terrain, i.e. temperature measurements depend on the altitude and exact position of the temperature sensors (Berger and Worlitschek, 2018). This complication including other factors such as the urban heat island effect or possible impact of other weather variables such as wind are neglected here.

Because hourly resolved temperature data is difficult to obtain with sufficient high spatial coverage, we calculate HDD by using daily minimum and maximum daily temperatures. We derive hourly values with the help of meteorological functions outlined in Day (2006). Alternative methods are available to calculate HDD such as using monthly mean air temperature and applying the Hitchin's formula (Department of the Environment, 1993).

To consider future weather and climate-related effects, we use the weather@home data presented in Guillod et al. (2017a). The weather@home data is obtained from a large number of simulations of the regional climate model HadRM3 run at a 50km grid resolution over the United Kingdom and Western Europe and presents different climate variables including minimum daily and maximum temperature. The large number of simulations makes the weather@home data suitable for studying extreme weather events. 100 different time series of the near future are downloaded from Guillod et al. (2017b). The temperature data is provided for a gridded representation across the United Kingdom for the year 2020 - 2049 for the Representative Concentration Pathway (RCP) RCP8.5 (Riahi et al., 2011). In order to simulate current weather, we use the provided simulation data for the year 2020. For assessing the impact of climate change, we use the data from this pathway, which represents high emissions and enables to highlight extreme changes to current climate. Compared to other pathways, this might however lead to overestimations. Because the applied energy demand modelling framework uses 365 days as an input, we expanded the provided 360 days to 365 days by duplicating 5 existing days evenly distributed across the year. For the simulation year 2050, we use the temperatures from the year 2049. For our modelling base year 2015, we calculated daily minimum and maximum daily temperatures from hourly resolved temperature data from the UK Met Office (2015) and mapped them to the spatial grid of the weather@ home data set. Because of using actual weather data for the base year, we include variability starting based on the weather@home data set only from the year 2020 onwards. Table 1 shows the average calculated annual, minimum and maximum daily heating and cooling degree days (CDD, HDD) across all regions.

Table 1: Calculated annual average, daily minimum and maximum heating and cooling degree days across all UK regions and 100 weather simulations. The weather simulations are taken from the RCP8.5 dataset (Riahi et al., 2011).

\begin{tabular}{|c|c|c|c|c|c|c|c|c|c|}
\hline & \multicolumn{3}{|c|}{ Current Weather (2020) } & \multicolumn{3}{|c|}{ Future Weather (2050) } & \multicolumn{3}{|c|}{ Change (\%) } \\
\hline & $\min$ & $\max$ & mean & $\min$ & $\max$ & mean & $\min$ & $\max$ & mean \\
\hline HDD & 1781 & 2377 & 2089 & 1335 & 1962 & 1600 & -25.1 & -17.4 & -23.4 \\
\hline CDD & 162 & 652 & 318 & 199 & 722 & 412 & 22.8 & 10.7 & 29.6 \\
\hline
\end{tabular}

\subsection{Energy demand projections for the United Kingdom}


We explore the uncertainty surrounding future energy demands based on a simulation approach. We project energy demands by defining four distinct scenarios simulating diverse futures (Figure 1).

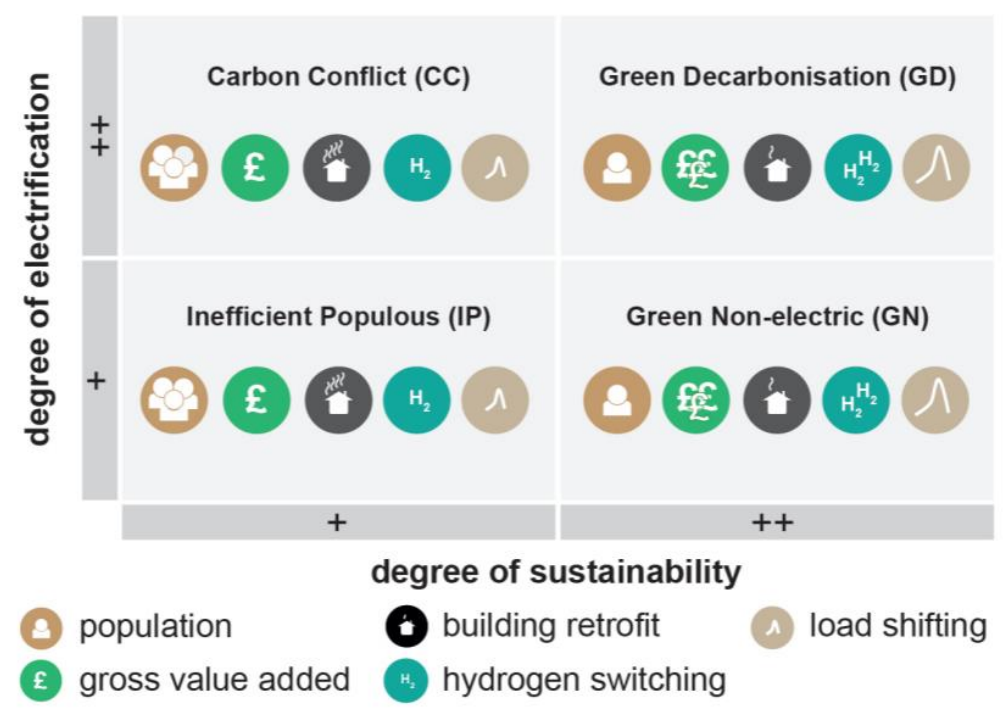

Figure 1: Overview of scenarios concerning the degree of electrification and degree of policy and technology innovation.

The scenarios differ most importantly with respect to the degree of electrification of heat and the degree of achieved overall sustainability measures. Electrification is expected to occur by the adoption of heat pumps for domestic space and water heating and by switching from gas to electricity in industry. The degree of policy and technology innovation represents how progressive and environmentally sustainable a scenario is with respect to the evolution of energy demand reduction efforts. Additionally, different types of uncertainties such as the level of hydrogen switching, load shifting capabilities, or the population development are defined across the scenarios (cf. Figure 1). Even though we only use four scenarios, they span a considerable scenario space as they combine factors either leading to overall higher or lower demands, i.e. the scenarios combine high population with low efficiency improvements (Carbon Conflict scenario) and vice-versa (Green Decarbonisation scenario). The scenarios can be summarised as follows:

- Under the Green Decarbonisation (GD), we see that a large-scale uptake in heat pumps and high-levels of substitution of natural gas with hydrogen are used to mitigate emissions in the industrial sector. We see low population growth and high reduction of heat loss, high efficiency improvements and load shifting capabilities.

- The Carbon Conflict (CC) scenario simulates the large-scale uptake of heat pumps for heating in a context with high population growth combined with little efficiency improvements. This scenario represents small overall reduction in heat loss of the building stock due to improvements in the building fabric as well as low levels of electrification of industrial processes and little switching to hydrogen.

- For the Inefficient Populous (IP) scenario, we see some uptake of heat pumps, but no largescale electrification of heating takes place. The low electrification occurs in a context with high population growth with small efficiency achievements. We see low 
levels of switching to hydrogen as an alternative fuel source or reducing heating demands due to improvements of the building stock.

- The Green Non-Electric (GN) scenario is a low electrification scenario combined with low population growth and high levels of sustainability. This scenario can be considered as the most sustainable one for our analysis due to achieved improved technological efficiencies, high reduction of heat losses and high levels of hydrogen switching combined with good load shifting capabilities.

A detailed overview of the parametrisation of the four scenarios considering different types of uncertainties is provided in Table 2. Socio-economic uncertainty is considered by selecting combined gross value added and population projections presented in Hall et al. (2016). Behavioural change related to heating behaviour is simulated by lowering the base temperature assumption. Load shifting capabilities for heating are simulated by assuming different levels on how much energy demand patterns can be changed by taking advantage of the thermal inertia of the building (Vanhoudt et al., 2014; Kreuder and Spataru, 2015; Heinen et al., 2017). For our scenarios, we assume different percentages on how much peak demand can be reduced due to load shifting. Efficiency improvements are defined for all end-uses which are not specified with technologies. To simulate potential efficiency improvements for end-uses with defined technologies, the achieved maximum efficiency level per technology is simulated (see Eggimann et al., 2018). With respect to hydrogen, there is now a consensus that complete decarbonisation of energy systems is not possible using only electricity (CCC, 2019), and that a carbon-free chemical vector, such a hydrogen, will be required for both some industrial processes (Philibert, 2017) and heating in some buildings (BEIS, 2018). We assume the introduction of hydrogen for heating and industrial processes from 2030 onwards. Whereas the large-scale deployment of hydrogen as a natural gas substitute will need considerable socio-technical changes, hydrogen can be safely injected into modern polyethylene gas distribution networks (Sadler et al 2016), and this has been confirmed in a demonstrator project (ITM Power 2020). A smart meter penetration is simulated by considering overall smart meter induced savings of $3 \%$ for service and residential end-uses (DECC, 2015). For the residential sector, all lighting is replaced by LED lighting by 2030 across all scenarios. Climate change is considered by using the temperature data set as outlined in Section 2.2 without considering further alternative climate scenarios.

These scenarios are intended to provide very different inputs for our assessment methodology in order to test implications of weather across different futures. It is however not meant to map out the full range of electricity demand futures, as the analysis concentrates on heating, so excludes demands from transport and cooling. Electricity demand is expected to be shaped by cooling demands as well, even though the extent of cooling demand and potential increase due to large-scale implementation of cooling devices in the United Kingdom may not be comparable to other places (Burillo et al., 2019). The recent heat wave in 2018 however shows that it is becoming increasingly important to include future cooling demands. Change in the uptake of cooling devices will however primarily impact peak summer electricity demand and are ignored for this analysis. Based on the change in simulated cooling degree days between 
current and future weather (see Table 1), we note on average an increase of $30 \%$ from 318 to 412 CDD (McNeil and Letschert, 2008). The maximum daily CDD increases by about $11 \%$. Based on the relationship between CDD and the uptake of cooling technologies, we thus expect an increase of total annual as well as peak cooling demand. According to data from the United States, such a change in CDD would lead to an expected increase of about $10 \%$ in the saturation of cooling technologies. Currently, the overall contribution of cooling to final energy demand is small $(<1 \%)$ (BEIS, 2018). Based on current usage, given than current and future cooling demand makes up such a small proportion of total electricity/energy demand, it was ignored for this paper. However, future behavioural and technological change could increase the installations of cooling technologies which means that investigating future cooling demand could play a role in understanding the total impacts to the electricity system. Similarly, electric vehicles are increasingly playing a decisive role in how much, and at which point in time, electricity is consumed. Electrifying transport is expected to increase overall power load on local distribution networks due to the large-scale diffusion of electric vehicles, playing a critical role with respect to load balancing and potential impacts on electricity peak (National Grid, 2017; Brady and O'Mahony, 2016). We however do not expect an increase in weather-based variability by including transport demands in this analysis as transportation is not directly linked to temperature. 
Table 2: Scenarios overview concerning the degree of electrification and degree of policy and technology innovation of the year 2050 .

\begin{tabular}{|c|c|c|c|c|c|}
\hline Model variables & Unit & GD & $\mathrm{CC}$ & GN & IP \\
\hline \multicolumn{6}{|l|}{ Uncertainty related to electrification } \\
\hline Heat pump penetration & $\%$ & 60 & 60 & 20 & 20 \\
\hline Electricity fuel switch in industry ${ }^{1}$ & $\%$ & 10 & 10 & 0 & 0 \\
\hline \multicolumn{6}{|l|}{ Socio-economic uncertainty } \\
\hline Population (mio) & people & 66.4 & 87.4 & 66.4 & 87.4 \\
\hline Gross value added (thousand per capita) & GBP & 37 & 32 & 37 & 32 \\
\hline \multicolumn{6}{|l|}{ Uncertainty related to heating } \\
\hline Hydrogen boiler penetration ${ }^{2}$ & $\%$ & 10 & 5 & 10 & 5 \\
\hline Thermal comfort & ${ }^{\circ} \mathrm{C}$ & 14.5 & 15.5 & 14.5 & 15.5 \\
\hline Heat loss improvement & $\%$ & 45 & 15 & 45 & 15 \\
\hline Heat recovered & $\%$ & 5 & 1 & 5 & 1 \\
\hline Load shifting $^{3}$ & $\%$ & 10 & 5 & 10 & 5 \\
\hline \multicolumn{6}{|l|}{ Other uncertainty } \\
\hline Hydrogen fuel switch in industry & $\%$ & 10 & 5 & 10 & 5 \\
\hline Residential efficiency improvement ${ }^{4}$ & $\%$ & 25 & 5 & 25 & 5 \\
\hline Service efficiency improvement ${ }^{5}$ & $\%$ & 20 & 2.5 & 20 & 2.5 \\
\hline Industry efficiency improvement ${ }^{6}$ & $\%$ & 25 & 5 & 25 & 5 \\
\hline Efficiency achievement level ${ }^{7}$ & $\%$ & 60 & 30 & 60 & 30 \\
\hline Smart meter penetration & $\%$ & 100 & 100 & 100 & 100 \\
\hline LED penetration $^{8}$ & $\%$ & 100 & 100 & 100 & 100 \\
\hline
\end{tabular}

${ }^{1}$ Gas is replaced with electricity in all industrial enduses

${ }^{2}$ Share of hydrogen based technology in total space and water heating

${ }^{3}$ Increase of electricity load factor for heating

${ }^{4}$ Affected enduses: consumer electronics, home computing

${ }^{5}$ Affected enduses: catering, ICT, small power, cooled storage, other

${ }^{6}$ Affected enduses: drying \& separation, motors, compressed air, lighting, refrigeration, other

${ }^{7}$ Achievement of theoretical efficiency levels for technologies (see Eggimann et al., 2019)

${ }^{8}$ Residential and service lighting

\section{Results and Discussion}

\subsection{National results}

National projected annual energy demands are calculated by aggregating across all simulated regions of the United Kingdom for gas, electricity and hydrogen (Figure 2) (simulation results data are available for download in Appendix A). From 2030 onwards, we see the introduction of hydrogen into the fuel mix. For electricity, considerable differences in overall electricity demands are simulated between the scenarios due to different level of fuel switching from gas to electricity, the degree of electrification of heat by more efficient heat pump technologies and other energy saving opportunities (see Table 2). As a result of this electrification, overall gas demand decreases. 


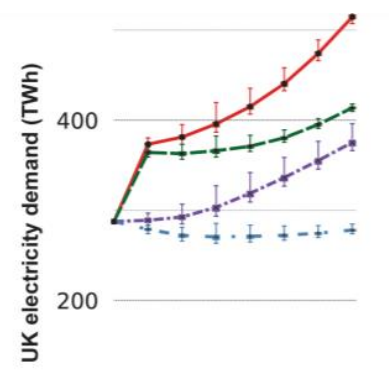

2020203020402050

Year

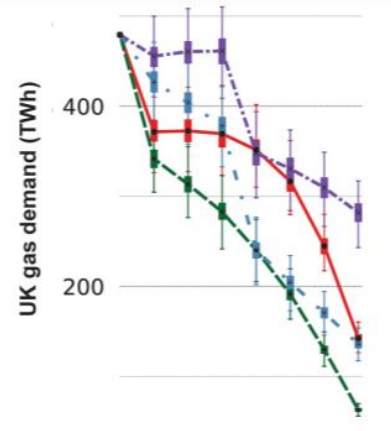

2020203020402050

Year

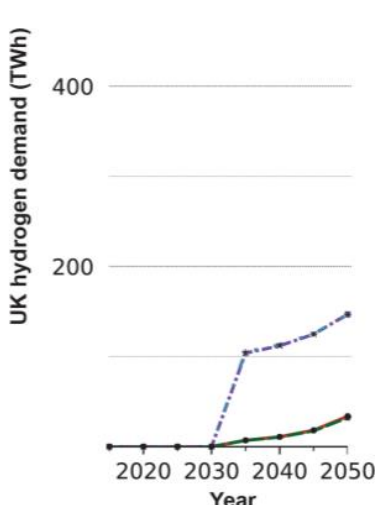

Year

Figure 2: Annual national energy demand per fuel type. Box plots show weather variability calculated based on 100 weather realisations for the year 2020 .

Weather effects due to cold weather are most crucial for analysing peak demands. While peak demand is often used for capacity expansion planning, variability is increasingly important to understand the need for reserve capacity and planning for operational aspects, including appropriate policy and technical measures such as demand response. Figure 3 a shows the simulated development in aggregated national electricity demand for the peak hour considering weather variability for all scenarios. We use the term weather variability for denoting the differences in simulated electricity demand across the 100 weather realisations.

For the carbon conflict scenario, having the largest projected increase of the mean peak demand, we project an increase of $43.5 \mathrm{GW}$ for the peak demand in the year 2050 compared to the base year peak. This shows the importance of investing in insulating the building stock before installing heat pumps. Whereas for this case, peak demand roughly doubles, peak demand decreases for the green non-electric scenario due to a low socio-economic development combined with high efficiency improvements and high load shifting capabilities for heating. Interestingly, the projected peak demands for the inefficient populous and green decarbonisation scenario for the year 2050 are comparable. This similar projection shows that not only the degree of electrification is crucial for projecting electricity peak demand but that we would expect effects in the same order due to socio-economic uncertainty. Even under a high electrification scenario with large uptake of energy saving opportunities, peak demands are projected to be similar to a low electrification scenario with high population growth and lower energy reducing efforts. Overall, we find that the differences resulting from socio-economic changes or the degree of electrification dominate the variation due to different weather scenarios. Whereas in relative terms, the impact of weather is small compared to the projected overall change in demand of annual or peak demand, in absolute power demands, the simulated range of weather-related impact on electricity peak demand is however considerable.

Considering the minimum and maximum simulated electricity peak demand, the impact of weather ranges across the scenarios for the year 2020 between $6.1-7.8 \mathrm{GW}(10.2-15.2 \%$ of mean peak demand) (Table 3). For the year 2050, the weather variability in peak electricity demand ranges between $6.2-14.6 \mathrm{GW}(9.8-22.2 \%$ of mean peak demand). We thus simulate an overall increase of weather variability from $2020-2050$. 

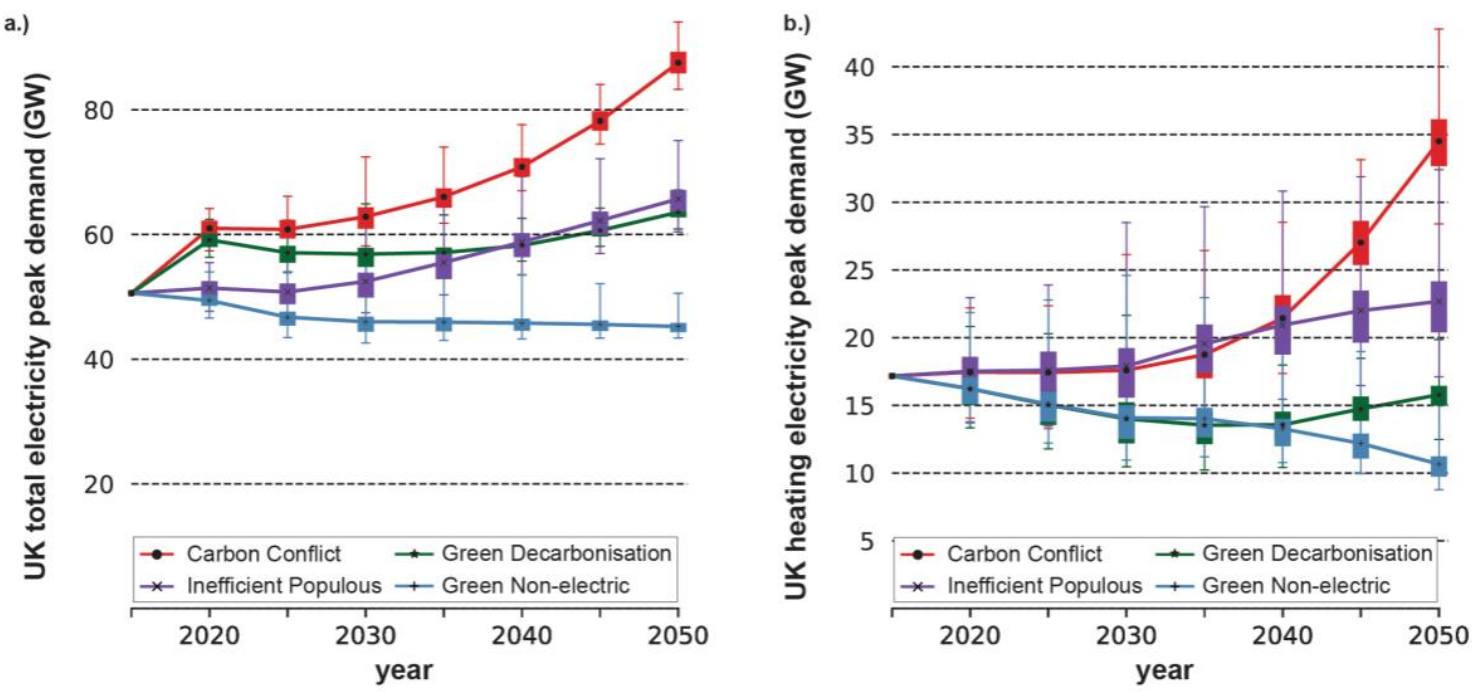

Figure 3: (a) National total peak electricity demand and (b) National electricity heating peak demand. Box plots show the weather-induced variability calculated based on 100 weather realisations of the year 2020 .

Across the scenarios, the various projected changes of heating and non-heating energy demands differ. In Figure 3b, the peak demand for heating energy demand and weather variability is shown on its own. We note in overall higher absolute electricity variability in case of higher absolute electricity demands for heating. The variability ranges for heating demand across the scenarios varies from 7.4-15.3 GW for the year 2050 (see Table 3). These simulation results show that with increased electrification, the (absolute) variability in electricity peak demand induced from exceptionally cold weather increases. Retrofitting the building stock would therefore reduce weather-related impacts at the same time as reducing overall demand. Also, our results suggest that alternative fuel sources such as hydrogen would reduce the demands on electricity generation and thus also reduce the risk of potential electricity disruption. Policies to reduce peak demand could thus lower network vulnerability and reduce economic damages resulting from network disruption (Zorn et al., 2020).

Our simulated results are in overall agreement with Staffell and Pfenninger (2018), who project daily variation of electricity peak demand based on 25 historic load profiles for the Two Degrees scenario from National Grid with a projected electricity peak of $62 \mathrm{GW}$ (National Grid, 2017) by 2030. This scenario corresponds conceptually to our Green Decarbonisation, where we project a lower average peak of $\sim 57 \mathrm{GW}$. For the year 2030, Staffell and Pfenninger (2018) simulated a mean peak hour demand of $\sim 60 \mathrm{GW}$ and a maximum simulated peak hour demand of $\sim 72 \mathrm{GW}$. For our Green Decarbonisation scenario, we simulate a mean peak hour demand of $57 \mathrm{GW}$ and a maximum peak of $65 \mathrm{GW}$. Our maximum and minimum electricity peak demand across our scenarios ranges for the year 2030 between 42.6-72.5 GW. For the year 2050, our simulated range between minimum and maximum peak demand is $43.4-94.1 \mathrm{GW}(-14.2 \%-+86.0 \%$ of the base year peak). We note that the impact of weather on overall gas demand is more pronounced as most gas is used for heating. Gas peak demand is however of lesser interest given its overall better storage capability compared to electricity. 
Table 3: Projected mean, minimum and maximum peak electricity demands in 2020 and 2050 for electricity heating demand and overall electricity demand (in GW) for 100 weather realisations.

\begin{tabular}{|c|c|c|c|c|c|}
\hline Scenario & mean peak & min peak & max peak & $\max -\min$ & $\%$ of mean \\
\hline \multicolumn{6}{|l|}{ Heating demand } \\
\hline Green Non-electric & 16.2 & 13.7 & 21.9 & 8.2 & 50.3 \\
\hline Inefficient Populous & 17.5 & 13.8 & 22.9 & 9.2 & 52.3 \\
\hline Green Decarbonisation & 16.2 & 13.3 & 20.8 & 7.5 & 46.1 \\
\hline Carbon Conflict & 17.5 & 14.1 & 22.2 & 8.1 & 46.6 \\
\hline \multicolumn{6}{|l|}{2050} \\
\hline Green Non-electric & 10.7 & 8.8 & 16.2 & 7.4 & 69.3 \\
\hline Inefficient Populous & 22.7 & 17.1 & 32.4 & 15.3 & 67.4 \\
\hline Green Decarbonisation & 15.8 & 12.5 & 19.9 & 7.4 & 46.8 \\
\hline Carbon Conflict & 34.5 & 28.4 & 42.8 & 14.4 & 41.7 \\
\hline \multicolumn{6}{|l|}{ Total demand } \\
\hline \multicolumn{6}{|l|}{2020} \\
\hline Green Non-electric & 49.4 & 46.6 & 54.0 & 7.4 & 15.0 \\
\hline Inefficient Populous & 51.4 & 47.7 & 55.5 & 7.8 & 15.2 \\
\hline Green Decarbonisation & 59.1 & 56.4 & 62.4 & 6.1 & 10.2 \\
\hline Carbon Conflict & 61.0 & 57.4 & 64.2 & 6.8 & 11.1 \\
\hline \multicolumn{6}{|l|}{2050} \\
\hline Green Non-electric & 45.2 & 43.4 & 50.6 & 7.2 & 15.8 \\
\hline Inefficient Populous & 65.7 & 60.4 & 75.1 & 14.6 & 22.3 \\
\hline Green Decarbonisation & 63.6 & 60.9 & 67.1 & 6.2 & 9.8 \\
\hline Carbon Conflict & 87.5 & 83.3 & 94.1 & 10.8 & 12.3 \\
\hline
\end{tabular}

\subsection{Regional results}

There are considerable regional differences in peak demand depending on population density, industry, the technological heating mix and the impacts of weather. Figure 4 shows the simulated regional effect of weather on national peak electricity demand in 2020 and 2050 assuming weather of the year 2020. Regional contributions to national hourly peak electricity demand for every region (shown on the x-axis) are plotted against the standard deviation of the regional contribution to national peak demand for all weather realisations ( $y$-axis). We find that the majority of local authority districts contribute to national electricity peak with less than $0.5 \mathrm{GW}$ with a standard deviation of below $0.02 \mathrm{GW}$. The few depicted outliers with drastic changes in demand result from using population scenarios having a few regions undergoing considerable change in population demands for the span of the simulated 45 years. 


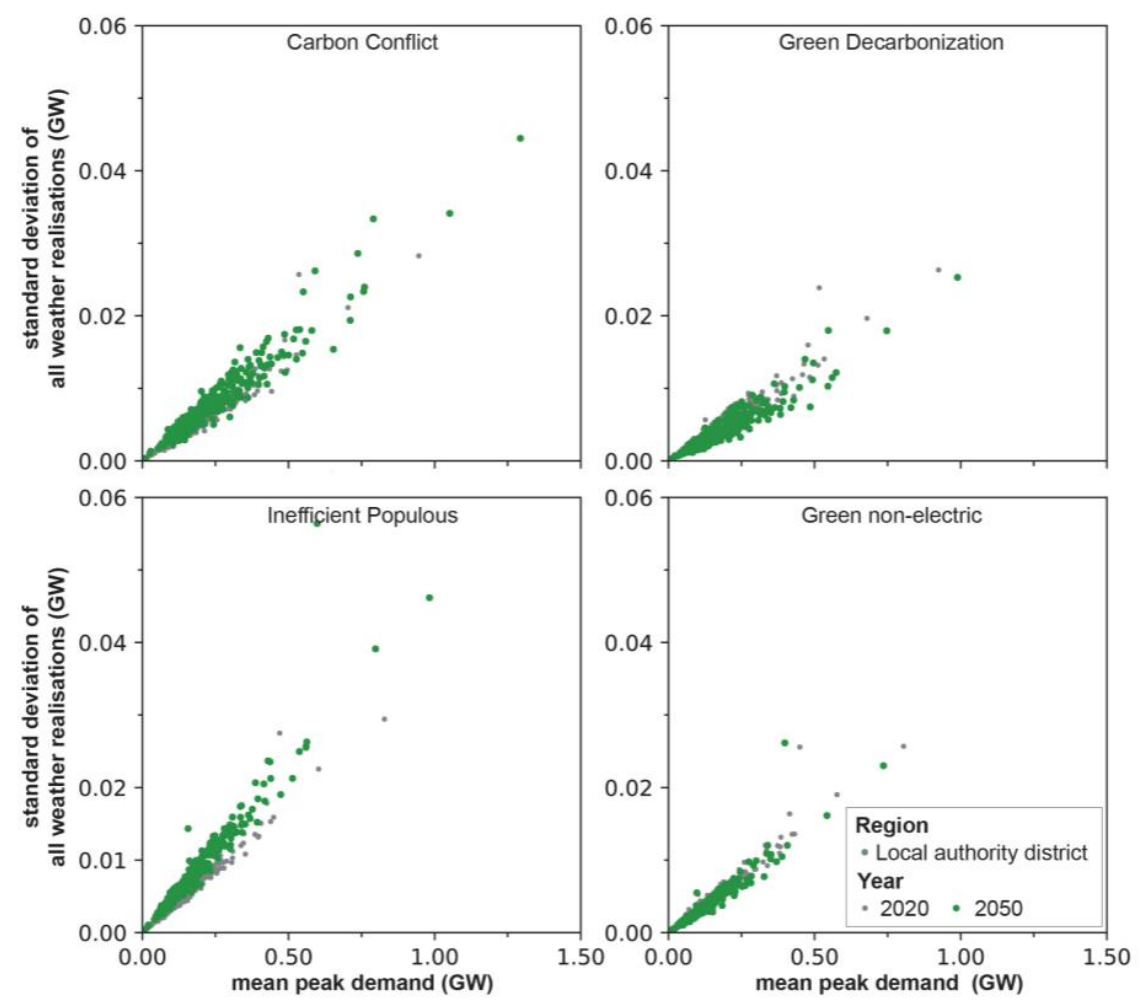

Figure 4: Contribution of local authority districts to national electricity peak and weather variability based on 100 weather realisations in 2020 and 2050 .

Figure 5 shows the projected change of regional contribution to peak and weather variability between 2020 - 2050 assuming weather of the year 2020. Based on changes in electricity peak demand and variability, four different categories of regions are classified. Regions with reduced overall demand and reduced variability (Category $C$ ) are of least priority as their energy infrastructure is expected to handle future energy demands projections well. Regions with higher variability but overall lower demands (Category D) are less critical as the variability is generally smaller compared to overall changes in demand. The most relevant regions are regions with increasing demand (Category B) and regions with a combined projected increase in demand and weather vulnerability (Category A). These regions are potential candidates for investment in future energy infrastructure or policy prioritisation to manage future supply and demand. These regions would profit most from measures to reduce weather related effects such as load shifting or reducing heating demands by retrofitting by insulation or other efficiency improvement methods. A geographical mapping of the classified regions is provided in Figure 6. The spatial mapping of the most critical regions in (Category A) reveals that particularly for the Inefficient Populous and Carbon Conflict scenario, special attention needs to be given to Southern England. We note that for the Green Decarbonisation scenario, i.e. a scenario with high electrification, the number of critical regions is much lower due to compensating increasing population with efficiency measures and improved load shifting capabilities. 

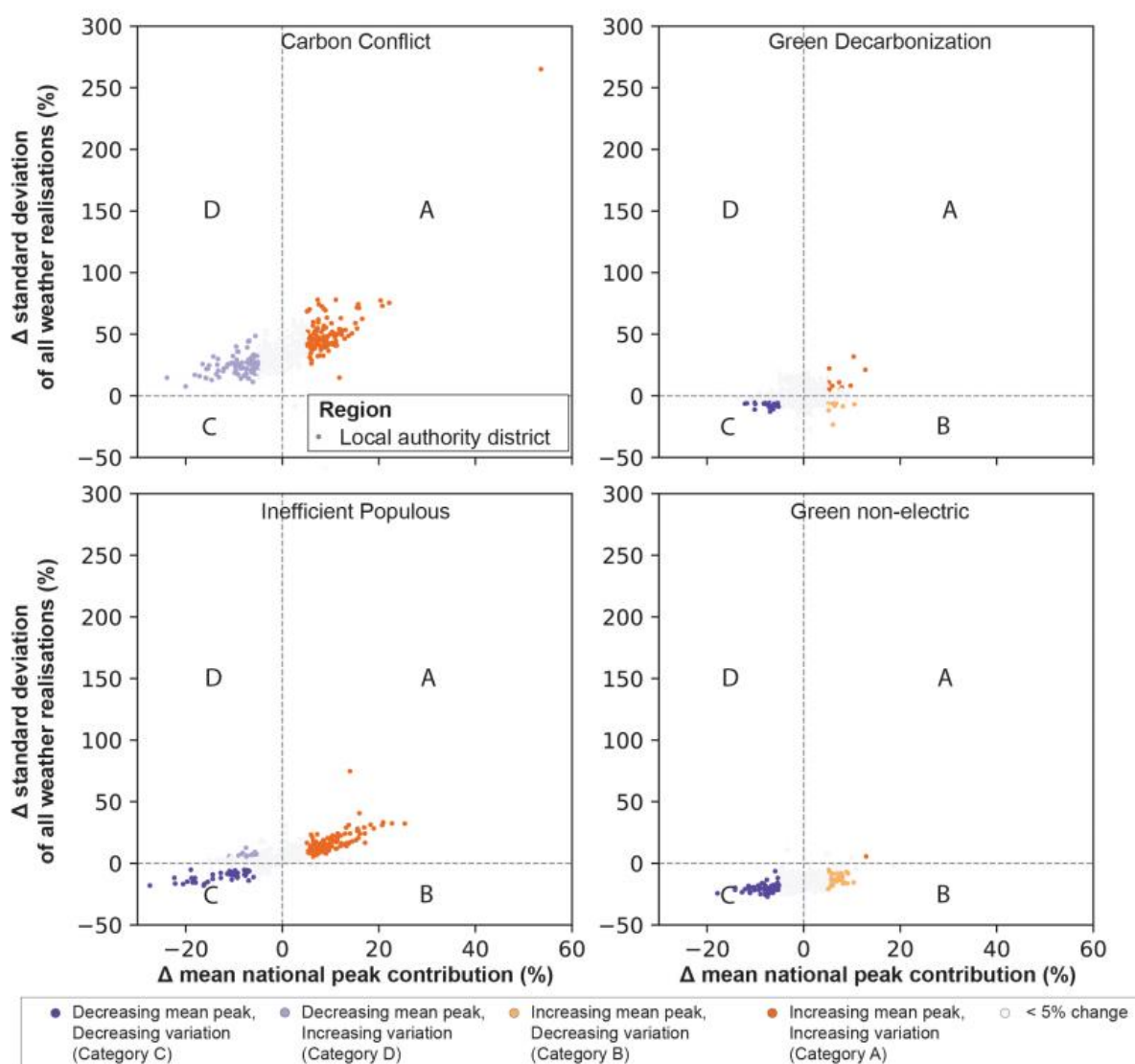

Figure 5: Change in contribution to national electricity peak demand per local authority district and weather variability for local authority districts based on 100 weather realisations of regional energy demands from between $2020-2050$. 


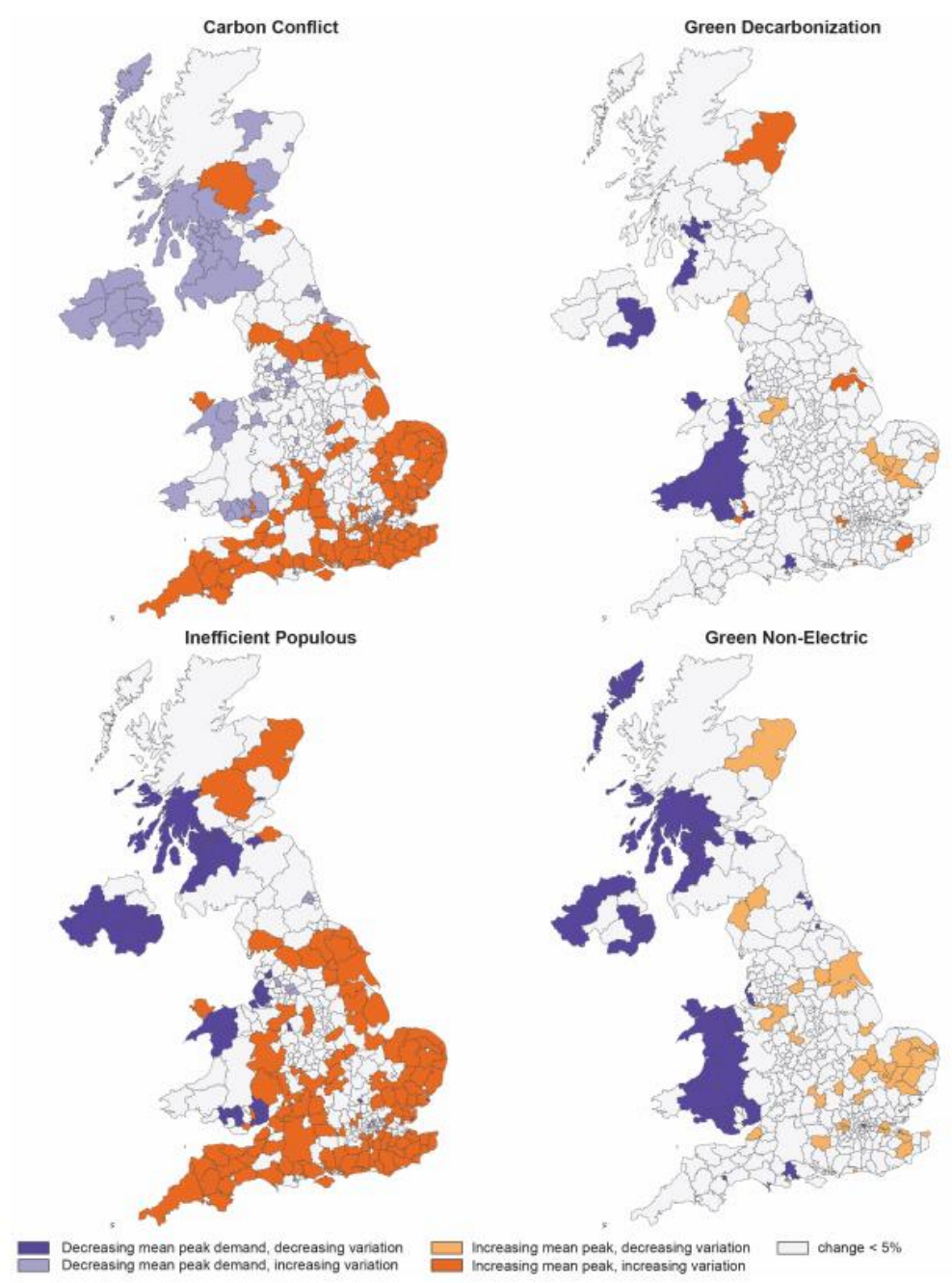

Figure 6: Geographical distribution of regions for each scenario classified according to increases in mean peak demand and weather-induced variability between 2020-2050. To highlight most sensible regions, regions with a smaller overall change in variability or peak demand than $5 \%$ are left blank.

\subsection{Impact of climate change}

So far, we have assessed the impact of weather by using simulation data from the year 2020 . In the following, we analyse weather variability for a different climate scenario by using the weather data provided by weather@ home of the Concentration Pathway RCP8.5 (see Section 2.2). We use the provided weather simulation from this pathway to illustrate as an example the impact of considerable changes to future weather. In case of other pathways simulating less pronounced changes to future weather, the simulated change is also expected to be less pronounced. Figure 7 shows the range of simulated peak electricity demands across all scenarios for current (2020) and future weather pattern taken from the RCP8.5. Using simulated future weather, the average peak decreases across all scenarios. For the two high-electrification scenarios, both the changes in peak demand (GD $+0.3 \%, \mathrm{CC}+1.8 \%)$ as well as changes to the weather-induced variability (range given as percentage of mean peak demand) increases (GD $+48.5 \%, \mathrm{CC}+18.6 \%)$. For the IP scenario, the lower variability might be explained by the heating saving effect due to warmer climate, which predominates the variability increase from electrification. For the GN scenario, we only see a small increase of variability of $4.6 \%$. 
We thus find that future weather may exacerbate the impact of electrification of heat on peak demand. However, we note that the socio-economic uncertainty across all scenarios predominates the weather as well as climate-induced variability.

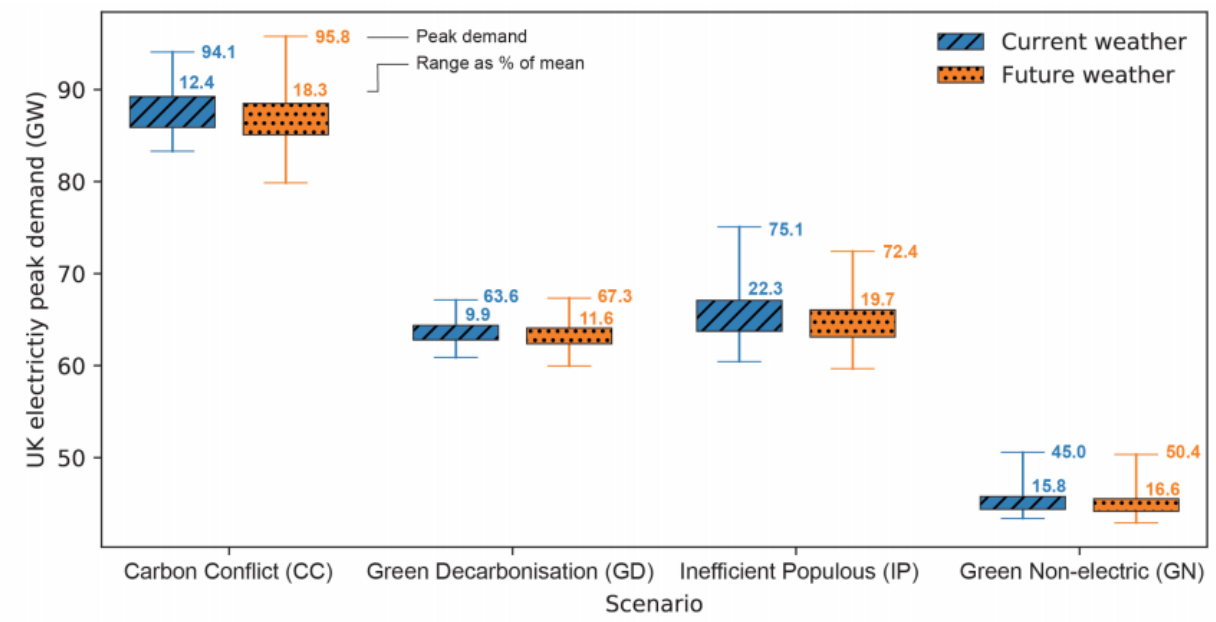

Figure 7: Comparison of simulated energy demands and weather-induced variability for 100 current (2020) and future (2050) weather realisations based on the RCP8.5 pathway.

\section{Conclusions}

In this analysis, we have shown how spatial and temporal high-resolution energy demand modelling enables the study of uncertainties surrounding future energy demands for heating and weather variability at a regional and hourly resolution. We have explored implications of decarbonizing heat by electrification on peak electricity demand and weather-induced variability for the United Kingdom based on 100 weather projections. Whereas the impact of weather on heating demand has predominantly been studied at a national level, this study maps regional weather effects including socio-technical uncertainties whilst projecting longterm energy demands changes. Future demand has been explored with four distinct scenarios considering different levels of electrification of heat and different levels of achieved energy demand reduction efforts.

We find that decarbonisation through electrification of heating will increase weather related impacts as heating is linked to temperature. With increasing absolute electricity demand for heating, the variability due to weather will increase if no additional measures are put in place to mitigate this effect. The effect of extreme weather on electricity demand could be reduced if heating is decarbonised with alternative fuels such as hydrogen, reducing the demand of electricity for heating. Similarly, we argue from the finding of this study, that reducing heating demand through retrofitting the building stock not only enables to lower peak demands but also reduces exposure to weather at the same time.

Based on the scenarios developed in this paper, we have found that the variability in annual energy demand arising from weather is much smaller than uncertainties related to future socio-economic development or the degree of electrification or fuel switching to alternative fuels such as hydrogen. For maximum power demand, however, the variation due to weather 
(assuming current weather patterns) has a considerable effect, ranging across the defined scenarios between $6.2-14.6 \mathrm{GW}(9.8-22.2 \%$ of mean peak demand) by 2050 . Using future weather simulation data reveals that climate change will exacerbate the impact of electrification of heat on peak demand. This change in impact of weather and the change in mean peak demand has clear implications for the electricity system operator who needs to anticipate potential changes to deliver power reliably across the network.

Projecting changes of peak demands and considering weather-induced variability are key for dimensioning energy systems and being able to pin-point places for implementing targeted demand side measures. We have mapped locations for prioritisation of implementation of load shifting or storage capabilities to reduce the influence of weather variability so as to better match energy supply and demand (see Figure 5). This is becoming particularly important in case of future more decentralised scenarios which depend less on electricity transmission and more on local supply. In particular, regions with high weather variability and high projected demand increase the need to be targeted for demand side measures and careful electrification of heat. This study also offers further insight into the policy question as to whether the focus should be laid on decarbonizing energy supply or reducing energy demands (Patt et al., 2018). Decarbonizing heat by electrification for the United Kingdom means moving away from gas. If the electrification of heat is not properly accompanied with reducing heating demand such as through investment in improving building energy efficiency, particularly for high population scenarios, this will lead to dramatic increases (about double) in electricity peak demand combined with increased exposure to weather effects at a national and regional level.

\section{Appendix A}

The simulation results are available for download online at http://dx.doi.org/10.17632/zszrfympwn.1. Fuel type specific energy demands are for download for every local authority district, projected years (2020, 2025, 2030, 2035, 2040, 2045 , 2050) and for each of the 8760 hours in a year assuming weather of the year 2020. Mean energy demands are provided across all weather realisations. Weather-induced variability on energy demand is additionally provided for mean demand \pm 2 standard deviations across the ensemble of 100 weather realisations.

\section{Acknowledgement}

We acknowledge Tom Russell for modelling support and data preparation. This work was supported by the UK Engineering and Physical Science Research Council under grant EP/N017064/1: MISTRAL: Multi-scale InfraSTRucture systems AnaLytics.

\section{[REFERENCES]}

